\title{
Generalized magnetic monopoles over contact manifolds
}

Mitsuhiro Itoh

Institute of Mathematics, University of Tsukuba, Ibanaki 305, Japan

(Received 13 May 1994; accepted for publication 20 June 1994)

A generalization of magnetic monopoles is given over an odd dimensional contact manifold and we discuss whether the Yang-Mills-Higgs functional attains at generalized monopoles the absolute minimal value, the topological invariant. (C) 1995 American Institute of Physics.

\section{REVIEW OF 3-DIM MAGNETIC MONOPOLES}

First we recall basic facts on 3-dim Yang-Mills-Higgs fields and magnetic monopoles. Let $P \rightarrow M$ be a $G$-principal bundle over a complete open oriented 3-dim Riemannian manifold $M$ ( $G$ is a compact semisimple group ). Let $(A, \Phi)$ be a smooth connection on $P$ and a smooth section of the adjoint bundle $\mathfrak{g}_{P}=P \times_{A d} \mathfrak{g}$, called a Higgs field. In what follows we call a pair $(A, \Phi)$ a configuration.

The Yang-Mills-Higgs functional $\mathscr{A}(A, \Phi)$ is defined as

$$
\mathscr{C}(A, \Phi)=\frac{1}{2} \int_{M}\left\{\left|F_{A}\right|^{2}+\left|D_{A} \Phi\right|^{2}\right\} d v_{g}
$$

We call a configuration Yang-Mills-Higgs field when the functional $\mathscr{A}$ is stationary at this configuration.

The Euler-Lagrange equations for the first variation of $\mathscr{A}_{B}$ are

$$
d_{A}\left(* F_{A}\right)+*\left[\Phi, D_{A} \Phi\right]=0, \quad d_{A}\left(* D_{A} \Phi\right)=0 .
$$

Here $F_{A}=d A+\frac{1}{2}[A \wedge A]$ is the curvature form of $A$, and $D_{A}, d_{A}$ are the covariant derivative and the covariant exterior derivative in the adjoint bundle $\mathfrak{g}_{P}$, respectively. Further $*$ denotes the Hodge star operator.

A configuration $(A, \Phi)$ satisfying the Bogomolny equation

$$
* F_{A}=\underline{1} D_{A} \Phi
$$

is called a (magnetic) monopole. It is easily verified by using the Bianchi identity and the Ricci identity that a monopole satisfies the Euler-Lagrange equations and hence is Yang-Mills-Higgs.

We take the special 3-manifold $M=\mathbf{R}^{3}$, the Euclidean 3-space and for simplicity the gauge group $G=\mathrm{SU}(2)$.

We consider configurations satisfying the asymptotical decay conditions at infinity of $\mathbf{R}^{3}$;

$$
\begin{gathered}
|\Phi|(x)=m+O(1 / r), \\
\left|F_{A}\right|(x), \quad\left|D_{A} \Phi\right|(x)=O\left(1 / r^{2}\right) .
\end{gathered}
$$

( $m$ is a constant, $r=|x|, x \in \mathbf{R}^{3}$ and $|\cdot|$ is the norm of the adjoint invariant inner product $(X, Y)$ $=-\operatorname{tr}(X Y)$ in the Lie algebra su(2)).

From the asymptotical conditions one gets a $C^{0}$ map $\Phi_{\infty}$ from the boundary of $\mathbf{R}^{3}$ at infinity, identified with a 2 -sphere $S^{2}$ of radius 1 , into a 2-sphere of radius $m$ in su(2) by

$$
\Phi_{\infty}(\hat{x})=\lim _{t \rightarrow \infty} \Phi(t \hat{x}), \quad \hat{x} \in S^{2}
$$


The map $\Phi_{\infty}$ has the mapping degree $k \in \mathbf{N}$, called a monopole charge of $(A, \Phi)$. The following shows that the Yang-Mills-Higgs functional attains under the asymptotical decay conditions the minimum represented by the topological invariant.

Proposition 1 (Refs. 1,2,3): For any configuration $(A, \Phi)$ of monopole charge $k$

$$
\mathscr{A}(A, \Phi)=4 \pi|k|+\frac{1}{2} \int_{M}\left|F_{A} \mp * D_{A} \Phi\right|^{2} d v_{g} \geqslant 4 \pi|k|
$$

and the equality holds if and only if $(A, \Phi)$ is a monopole.

\section{GENERALIZATION OF MAGNETIC MONOPOLES}

Yang-Mills-Higgs fields can be defined over a complete open manifold of an arbitrary dimension. Indeed a Yang-Mills-Higgs field is defined, same as in the 3-dimensional case, as a stationary point of the Yang-Mills-Higgs functional (1). So the equations (2) are valid also as the Euler- Lagrange equations for arbitrary dimensional Yang-Mills-Higgs fields.

We consider a generalization of 3-dim monopole over a manifold of an arbitrary odd dimension admitting a special geometrical structure. Here a generalization should be canonically given in the sense that (i) the equation for generalized monopoles is a first order equation, like the Bogomolny equation (3) and (ii) the generalized monopoles reduce to the original 3-dim monopoles, when the manifold dimension is 3.

Let $M$ be a complete open oriented Riemannian manifold of dimension $2 n+1$. We call $M$ a contact manifold if $M$ has a 1 -form $\eta$ such that $2 n+1-$ form $\eta \wedge(d \eta)^{n}$ is nonzero over $M . \eta$ is called a contact form.

Set $\omega=d \eta$. $\omega$ is a closed 2 -form.

Definition: Let $P \rightarrow M$ be a $G$-principal bundle over a complete open contact manifold $M$. A configuration $(A, \Phi)$ on $P$ is called a generalized monopole if $(A, \Phi)$ satisfies the generalized Bogomolny equations

$$
* F_{A}=c D_{A} \Phi \wedge \omega^{n-1}, \quad * D_{A} \Phi=c F_{A} \wedge \omega^{n-1}
$$

( $c$ is a constant). It is clear that when $\operatorname{dim} M=3(8)$ reduces to the single equation (3) which is free from any contact form on $M$.

Proposition 2: A generalized monopole is Yang-Mills-Higgs.

Proof: It suffices to check (2). Set $\Omega=\omega^{n-1}$. Then

$$
\begin{aligned}
d_{A}\left(* F_{A}\right)+*\left[\Phi, D_{A} \Phi\right] & =c d_{A}\left(D_{A} \Phi \wedge \Omega\right)+\left[\Phi, c F_{A} \wedge \Omega\right] \\
& =c d_{A} D_{A} \Phi \wedge \Omega-c D_{A} \Phi \wedge d \Omega+c\left[\Phi, F_{A} \wedge \Omega\right] \\
& =c\left[F_{A}, \Phi\right] \wedge \Omega+c\left[\Phi, F_{A}\right] \wedge \Omega \\
& =0 .
\end{aligned}
$$

Similarly

$$
d_{A}\left(* D_{A} \Phi\right)=d_{A}\left(c F_{A} \wedge \Omega\right)=c\left(d_{A} F_{A} \wedge \Omega+F_{A} \wedge d \Omega\right)=0
$$

So any generalized monopole is Yang-Mills-Higgs.

The possible values the constant $c$ of (8) takes depend only on the dimension of $M$, as will be shown in 3. 
We consider next the question whether the thus defined generalized monopoles take the absolute minimal value for the functional 8 .

Same as before, we assume that the structure group $G$ is $S U(2)$.

It is easy to show the following identity:

$$
\begin{aligned}
& \left\{\left|F_{A}-c *\left(D_{A} \Phi \wedge \Omega\right)\right|^{2}+\left|D_{A} \Phi-c *\left(F_{A} \wedge \Omega\right)\right|^{2}\right\} d v \\
& \quad=\left\{\left|F_{A}\right|^{2}+\left|D_{A} \Phi\right|^{2}+c^{2}\left|F_{A} \wedge \Omega\right|^{2}+c^{2}\left|D_{A} \Phi \wedge \Omega\right|^{2}\right\} d v-4 c\left((-\operatorname{tr})\left(F_{A} \wedge D_{A} \Phi\right)\right) \wedge \Omega
\end{aligned}
$$

The $2 n+1$-form in the last term is an exact form, namely,

$$
\left(-\operatorname{tr}\left(F_{A} \wedge D_{A} \Phi\right)\right) \wedge \Omega=d \Theta
$$

where $\Theta$ is a $2 n$-form given by $\Theta=-\operatorname{tr}\left(F_{A} \cdot \Phi\right) \wedge \Omega$.

The integral $\int_{M}\left(-\operatorname{tr}\left(F_{A} \wedge D_{A} \Phi\right)\right) \wedge \Omega=\int_{M} d \Theta$ is shown to be a topological invariant determined by the Higgs field $\Phi_{\infty}$ at infinity, provided certain asymptotical conditions, one on $M$ and another on configurations, are fulfilled. Suppose ( $\downarrow 1$ ) there is $a_{o}>0$ such that the distance function from a point $o \in M \quad x \in M \mapsto d(x, o)$ has non zero gradient vector for all $x$ of $d(x, o) \geqslant a_{o}$ and hence for all sufficiently large $a \partial M_{a}=\{x ; d(x, o)=a\}$ is a smooth hypersurface in $M$ smoothly parametrized by $a,(\hbar 2)(A, \Phi)$ is of finite $\mathscr{A}(A, \Phi)$ and satisfies with respect to their restriction to $\partial M_{a}$

$$
|\Phi|(x)=m+O(1 / a), \quad\left|D_{A} \Phi\right|(x)=O\left(1 / a^{2}\right)
$$

$(a=d(x, o))$.

Integrating (9) over $M$, we get

$$
\begin{aligned}
& \int_{M}\left\{\left|F_{A}\right|^{2}+\left|D_{A} \Phi\right|^{2}+c^{2}\left|F_{A} \wedge \Omega\right|^{2}+c^{2}\left|D_{A} \Phi \wedge \Omega\right|^{2}\right\} \\
& =\int_{M}\left\{\left|F_{A}-c *\left(D_{A} \Phi \wedge \Omega\right)\right|^{2}+\left|D_{A} \Phi-c *\left(F_{A} \wedge \Omega\right)\right|^{2}\right\}+4 c \int_{M} d \Theta .
\end{aligned}
$$

Here $\int_{M} d \Theta=\lim _{a \rightarrow \infty} \int_{d(x, o) \leqslant a} d \Theta$ and the integral $\int_{d(x, o) \leqslant a} d \Theta$ reduces by Stoke's theorem to the hypersurface integral $\int_{\partial M_{a}} \Theta$ to which we are able to use the conditions ( $\left.i v 1\right),(i 2)$ and apply the argument given in Horváthy and Rawnsley ${ }^{4}$ and II.5, Jaffe and Taubes. ${ }^{1}$ Therefore $\int_{M} d \Theta$ turns out to be a topological invariant of the Higgs field $\Phi_{\infty}$ at infinity, which we denote by $p\left(\Phi_{\infty}\right)$.

Proposition 3: Let $M$ be a complete open oriented Riemannian $2 n+1$-dim manifold having a contact form $\eta$. Then the following inequality holds for any configuration $(A, \Phi)$ under the conditions ( $\lesssim 1)$, ( 252 ).

$$
\int_{M}\left\{\left|F_{A}\right|^{2}+\left|D_{A} \Phi\right|^{2}+c^{2}\left|F_{A} \wedge \Omega\right|^{2}+c^{2}\left|D_{A} \Phi \wedge \Omega\right|^{2}\right\} \geqslant 4 c p\left(\Phi_{\infty}\right)
$$

and the equality holds if and only if $(A, \Phi)$ is a generalized monopole.

Proof: The inequality clearly follows from (11).

Suppose that the equality in (12) holds. Then

$$
F_{A}=c *\left(D_{A} \Phi \wedge \Omega\right), \quad D_{A} \Phi=c *\left(F_{A} \wedge \Omega\right)
$$


Since $\operatorname{dim} M$ is odd, the star operator $*$ for 1 - and 2 -forms satisfies $* \circ *=i d$ so that the above equations are just (8).

\section{CONTACT MANIFOLDS AND ASSOCIATED CONTACT METRICS}

A contact manifold $M$ with a contact form $\eta$ is endowed with a metric $g$ associated to the contact form (see Proposition in Sec. 3, Ref. 5).

In fact, the contact form $\eta$ yields on $M$ a contravariant vector field $\xi$ and a tensor field $\varphi$ of type $(1,1)$ satisfying

$$
\eta(\xi)=1, \quad \varphi(\varphi(X))=-X+\eta(X) \xi
$$

( $X$ is an arbitrary tangent vector).

Then $M$ has a metric $g$ which is compatible with the $\eta$, namely,

$$
g(\varphi(X), \varphi(Y))=g(X, Y)-\eta(X) \eta(Y)
$$

and further satisfies

$$
d \eta(X, Y)=g(X, \varphi(Y))
$$

We call such a metric $g$ an associated contact metric.

Example: $\mathbf{R}^{2 n+1}$ is a contact manifold with a contact form $\eta=d z-\Sigma_{i} y^{i} d x^{i}$ in terms of the Cartesian coordinates $\left\{x^{i}, y^{i}, z\right\} . \omega=d \eta=\Sigma d x^{i} \wedge d y^{i}$. The metric $g=\eta \otimes \eta+\Sigma\left\{\left(d x^{i}\right)^{2}+\left(d y^{i}\right)^{2}\right\}$ is an associated complete contact metric on $\mathbf{R}^{2 n+1}$.

We remark that the Euclidean metric for $2 n+1>3$ cannot be associated to any contact form (see Theorem, Chap VI, Ref. 5). This fact may be consistent with that any Yang-Mills-Higgs field of,$\ell<\infty$ on the Euclidean space $\mathbf{R}^{\prime}, \ell>3$, turns out trivial, namely, a flat connection with a covariant constant Higgs field( see the argument of the stress tensor in Chap. II, Ref. 1).

Suppose that an open contact manifold $M$ with a contact form $\eta$ admits a complete associated contact metric $g$.

To investigate the absolute minimal value of the functional $\mathscr{A}$ on $M$ we define the operator over $p$-forms on $M$

$$
\text { *o } L: \Lambda^{p}(M) \rightarrow \Lambda^{3-p}(M),
$$

where $L: \Lambda^{p}(M) \rightarrow \Lambda^{2 n+p-2}(M)$ is the exterior multiplication by the $2 n-2$-form $\Omega$. So one defines an endomorphism of $\left(\Lambda^{1} \oplus \Lambda^{2}\right)(M)$

$$
(* \circ L)(\alpha, \beta)=((* \circ L)(\beta),(* \circ L)(\alpha)), \quad(\alpha, \beta) \in\left(\Lambda^{1} \oplus \Lambda^{2}\right)(M) .
$$

As is easily shown, this endomorphism is self adjoint with respect to the naturally defined metric on $\left(\Lambda^{1} \oplus \Lambda^{2}\right)(M)$.

Lemma: Let $(M, \eta, g)$ be a contact manifold of dimension $2 n+1>3$ with an associated contact metric.

Then $(* \circ L)^{2}$ has the eigenvalues $0,\{(n-1) !\}^{2}, n\{(n-1) !\}^{2}$ so that the endomorphism $* \circ L$ has eigenvalues $0, \pm(n-1) !$ and $\pm(n-1) ! \sqrt{n}$. Furthermore for any $(\alpha, \beta) \in\left(\Lambda^{1} \oplus \Lambda^{2}\right)(M)$

$$
|\alpha \wedge \Omega|^{2}+|\beta \wedge \Omega|^{2} \leqslant n\{(n-1) !\}^{2}\left(|\alpha|^{2}+|\beta|^{2}\right)
$$

Here the equality holds in (18) if and only if $(\alpha, \beta)$ is in the eigenspace belonging to eigenvalue $n\{(n-1) !\}^{2}$. 
Proof: The first part of the lemma is an elementary exercise in Grassmannian algebra, provided we use an associated local orthonormal basis $\left\{\xi, e_{2 i-1}, e_{2 i}=\varphi\left(e_{2 i-1}\right)\right\}$ and its dual basis $\left\{\eta, \theta^{2 i-1}, \theta^{2 i}\right\}$.

To see (18) we write

$$
|\alpha \wedge \Omega|^{2}+|\beta \wedge \Omega|^{2}=|(* \circ L) \alpha|^{2}+|(* \circ L) \beta|^{2}
$$

from which the desired inequality is available.

Remark: The eigenspaces of $* \circ L$ have the dimension $(n-1)(2 n+1)$ for eigenvalue $0,2 n$ for eigenvalue $\pm(n-1) !$ and 1 for $\pm(n-1) ! \sqrt{n}$, respectively.

In fact, $(\eta, \pm \omega)$ give the eigenvectors of eigenvalues $\pm(n-1) ! \sqrt{n}$ and $\left\{\left(\theta^{2 i-1}, \pm \theta^{2 i} \wedge \eta\right)\right.$, $\left.\left(\theta^{2 i}, \pm \eta \wedge \theta^{2 i-1}\right)\right\}_{i=1, \ldots, n}$ form bases of the eigenspaces of eigenvalues $\pm(n-1)$ !.

Moreover the eigenspace of zero eigenvalue, identified with $\operatorname{Ker} L$ in $\Lambda^{2}(M)$, has the following basis

$$
\begin{gathered}
\theta^{1} \wedge \theta^{2}-\theta^{2 i-1} \wedge \theta^{2 i}, \quad 2 \leqslant i \leqslant n, \\
\theta^{2 i-1} \wedge \theta^{2 j-1}, \quad \theta^{2 i-1} \wedge \theta^{2 j}, \theta^{2 i} \wedge \theta^{2 j-1}, \quad \theta^{2 i} \wedge \theta^{2 j}, \quad 1 \leqslant i<j \leqslant n .
\end{gathered}
$$

Note for $M$ of dimension $3 * \circ L$ has only eigenvalues $\pm 1 . \quad\left(\eta, \pm \omega= \pm \theta^{1} \wedge \theta^{2}\right)$, $\left(\theta^{1}, \pm \theta^{2} \wedge \eta\right)$ and $\left(\theta^{2}, \pm \eta \wedge \theta^{1}\right)$ form bases of the eigenspaces of eigenvalues \pm 1 , respectively.

Further the generalized Bogomolny equations (8) can be written as

$$
\left(D_{\Lambda} \Phi, F_{A}\right)=c(* \circ L)\left(D_{A} \Phi, F_{A}\right)
$$

in terms of the endomorphism $* \circ L$. So, a generalized monopole $(A, \Phi)$ must belong to the eigenspace of $* \circ L$ with eigenvalue $c^{-1}$ and hence the possible values of the constant $c$ in (8) are $\pm 1 /(n-1) !$ and $\pm 1 /(n-1) ! \sqrt{n}$.

The following is an immediate consequence of Proposition 3 and the above lemma.

Proposition 4: Under the conditions same as in Proposition 3

$$
\mathscr{A}(A, \Phi) \geqslant \frac{2 c}{1+a_{n}^{2} c^{2}} p\left(\Phi_{\infty}\right)
$$

$\left(a_{n}=(n-1) ! \sqrt{n}\right)$. Here the equality holds if and only if a configuration $(A, \Phi)$ is a generalized monopole of the constant $c= \pm a_{n}^{-1}$.

Proof: Although we have shown the proposition, we will give another inequality on $A$, which is quite parallel to that for the generalized (anti-)self-dual connections on a quaternionic Kähler manifold. ${ }^{6}$

Decompose the adjoint bundle valued forms $\Xi=\left(D_{A} \Phi, F_{A}\right)$ as the sum

$$
\Xi=\Xi_{1}+\Xi_{2}+\Xi_{-1}+\Xi_{-2}+\Xi_{0}
$$

where $\Xi_{ \pm 1}, \Xi_{ \pm 2}$ and $\Xi_{0}$ are the components of $\Xi$ corresponding to eigenvalues $\pm(n-1) ! \sqrt{n}$, $\pm(n-1)$ ! and 0 , respectively.

The topological invariant $p\left(\Phi_{\infty}\right)$ is then represented as

$$
p\left(\Phi_{\infty}\right)=\frac{1}{2}\left\{a_{n}\left|\Xi_{1}\right|^{2}+b_{n}\left|\Xi_{2}\right|^{2}-a_{n}\left|\Xi_{-1}\right|^{2}-b_{n}\left|\Xi_{-2}\right|^{2}\right\},
$$

$\left(b_{n}=(n-1) !<a_{n}\right)$ because by using the inner products we can write

$$
\left(-\operatorname{tr}\left(F_{A} \wedge D_{A} \Phi\right)\right) \wedge \Omega=\left(F_{A}, * \circ L\left(D_{A} \Phi\right)\right)=\left(D_{A} \Phi, * \circ L\left(F_{A}\right)\right) .
$$


On the other hand the functional $t \&$ has the form

$$
\mathscr{A}(A, \Phi)=\frac{1}{2}\left\{\left|\Xi_{1}\right|^{2}+\left|\Xi_{2}\right|^{2}+\left|\Xi_{-1}\right|^{2}+\left|\Xi_{-2}\right|^{2}+\left|\Xi_{0}\right|^{2}\right\}
$$

so that from (2I)

$$
. \not(A, \Phi)=\frac{1}{a_{n}} p\left(\Phi_{\infty}\right)+\frac{1}{2}\left\{\left(1-\frac{b_{n}}{a_{n}}\right)\left|\Xi_{2}\right|^{2}+2\left|\Xi_{-1}\right|^{2}+\left(1+\frac{b_{n}}{a_{n}}\right)\left|\Xi_{-2}\right|^{2}+\left|\Xi_{0}\right|^{2}\right\}
$$

from which it follows that a $c$-generalized monopole, $c=1 / a_{n}$, minimizes the functional $\mathscr{A}$. Similarly, a $c$-generalized monopole, $c=-1 / a_{n}$, also minimizes.

In spite of the above characterization of $\pm a_{n}^{-1}$-generalized monopole the following proposition shows that when $\operatorname{dim} M>3$ there do not exist any $\pm\left(1 / a_{n}\right)$-generalized monopoles with nonzero topological invariant satisfying ( $\leqslant 2$ ).

Proposition 5: Let $(A, \Phi)$ be a $\pm\left(1 / a_{n}\right)$-generalized monopole satisfying ( $\left.\longleftarrow 2\right)$. If $\operatorname{dim} M>3$, then $(A, \Phi)$ must be a trivial configuration, that is, $F_{A}=0$ and $D_{A} \Phi=0$, and hence the topological invariant $p\left(\Phi_{\infty}\right)=0$.

Proof: Since the eigenspaces of eigenvalues $\pm\left(1 / a_{n}\right)$ are $\mathbf{R}( \pm \eta, \omega)$, it holds $\left(D_{A} \Phi, F_{A}\right)=\Psi \otimes( \pm \eta, \omega)$ for some section $\Psi$ of $\mathfrak{g}_{P}$. It follows from the Bianchi identity that $D_{A} \Psi=0$. So the norm of $D_{A} \Phi= \pm \Psi \otimes \eta$ is constant which must be zero from ( $\left.\downarrow 2\right)$. Therefore $\Psi=0$, i.e., the pair $\left(D_{A} \Phi, F_{A}\right)=0$. The invariant $p\left(\Phi_{\infty}\right)=\int_{M}\left(-\operatorname{tr}\left(F_{A} \wedge D_{A} \Phi\right)\right) \wedge \Omega$ now becomes zero.

\section{HERMITIAN GEOMETRY OF GENERALIZED MONOPOLE}

Let $M$ be a contact manifold with the respective tensor fields $\eta, \xi, \varphi, g$ defined at 3 . The product manifold $M \times \mathbf{R}$ (or $M \times S^{1}$ ) then has the almost Hermitian structure, that is, admits an almost complex structure $J$,

$$
J\left(X+f \frac{d}{d t}\right)=\varphi(X)-f \xi+\eta(X) \frac{d}{d t}
$$

and a Hermitian metric $\bar{g}$,

$$
\bar{g}\left(X+f_{1} \frac{d}{d t}, Y+f_{2} \frac{d}{d t}\right)=g(X, Y)+f_{1} \cdot f_{2} .
$$

Every configuration $(A, \Phi)$ on $M$ is then regarded as a time-independent connection $\mathbf{A}=A+\Phi d t$ on $M \times \mathbf{R}$.

At each point of $M \times \mathbf{R}$ the space of 2 -forms $\Lambda^{2}(M \times \mathbf{R})$ can be identified as

$$
\Lambda^{2}(M \times \mathbf{R}) \cong\left(\Lambda^{1} \oplus \Lambda^{2}\right)(M)
$$

by

$$
\alpha \wedge d t+\beta \rightarrow(\alpha, \beta)
$$

Then $(\alpha, \beta) \in\left(\Lambda^{1} \oplus \Lambda^{2}\right)(M)$ satisfies $(\alpha, \beta)=c(* \circ L)(\alpha, \beta)$, if and only if

$$
\xi(\alpha \wedge d t+\beta)=c(\alpha \wedge d t+\beta) \wedge \Omega
$$

where $i$ is the Hodge star operator on $M \times \mathbf{R}$ and the $(2 n-2)$-form $\Omega=\omega^{n-1}$ is considered as a form over $M \times \mathbf{R}$. This is directly derived from the following 


$$
\text { is: } \Lambda^{2}(M \times \mathbf{R}) \rightarrow \Lambda^{2 n}(M \times \mathbf{R}) ; \quad \text { is }(\alpha \wedge d t)=* \alpha, \quad \succsim \beta=(* \beta) \wedge d t
$$

Since the curvature form $F_{\mathbf{A}}$ of $\mathbf{A}$ is

$$
F_{\mathbf{A}}=D_{A} \Phi \wedge d t+F_{A}
$$

by using (29) and (30) we have obviously

Proposition 6: Let $(A, \Phi)$ be a configuration on $M$.

(i) $(A, \Phi)$ is a Yang-Mills-Higgs field on $M$ if and only if $\mathbf{A}$ is a Yang-Mills connection on the almost Hermitian manifold $M \times \mathbf{R}$.

(ii) $(A, \Phi)$ is a generalized monopole with constant $c$ if and only if $\mathbf{A}$ satisfies the equation

$$
\lesssim F_{\mathrm{A}}=c F_{\mathrm{A}} \wedge \omega^{n-1}
$$

The equation (32) is quite similar to the Kähler manifold version of (anti-)self-dual equation, ${ }^{7,8}$ whereas in our case $\omega$ is a degenerate 2 -form. When $\operatorname{dim} M=3$ the proposition gives us the classical observation given in Manton $^{9}$ that $(A, \Phi)$ is a Yang-Mills-Higgs field (a monopole) if and only if $\mathbf{A}$ is a Yang-Mills connection (an instanton ).

Let $\tilde{\omega}=d\left(e^{-t} \eta\right)$ be an exact 2-form on $M \times \mathbf{R}$. Then $\tilde{\omega}=e^{-t}(\eta \wedge d t+\omega)$ is the fundamental form of the Hermitian metric $e^{-t} \bar{g}$. Note that $\tilde{\omega}^{n-1}=e^{-(n-1) t}\left(\omega^{n-1}+(n-1) \omega^{n-2} \wedge \eta \wedge d t\right)$.

Since for a $\bar{g}$-orthonormal basis $\left\{\theta^{i}, \eta, d t\right\}$ on $M \times \mathbf{R}$ we have

$$
\left(\theta^{2 i-1}+\sqrt{-1} \theta^{2 i}\right) \wedge(\eta+\sqrt{-1} d t)=-\left(\theta^{2 i} \wedge d t+\eta \wedge \theta^{2 i-1}\right)+\sqrt{-1}\left(\theta^{2 i-1} \wedge d t+\theta^{2 i} \wedge \eta\right)
$$

and

$$
\left(\theta^{2 i-1}-\sqrt{-1} \theta^{2 i}\right) \wedge(\eta+\sqrt{-1} d t)=\left(\theta^{2 i} \wedge d t+\eta \wedge \theta^{2 i-1}\right)+\sqrt{-1}\left(\theta^{2 i-1} \wedge d t+\theta^{2 i} \wedge \eta\right)
$$

so that (28) gives from the remark in 3 a characterization of $\pm\{(n-1) !\}^{-1}$-generalized monopole in terms of Hermitian geometry ${ }^{8,10}$ as

Proposition 7: (i) $(A, \Phi)$ is a $\pm\{(n-1) !\}^{-1}$-generalized monopole on $M$ if and only if $F_{\mathrm{A}}$ of $A$ has only components of the form taken by the real or imaginary part of $\left(\theta^{2 i-1} \pm \sqrt{-1} \theta^{2 i}\right)$ $\wedge(\eta+\sqrt{-1} d t)$.

(ii) Therefore as a $\mathfrak{g}_{p}$-valued 2 -form the curvature form $F_{\mathrm{A}}$ for a $\{(n-1) !\}^{-1}$-generalized monopole has no $(1,1)$ - components.

(iii) Further $F_{\mathrm{A}}$ for $-\{(n-1) !\}^{-1}$-generalized monopole is a primitive $(1,1)$-form, i.e., a $(1,1)$-form orthogonal to the $\tilde{\omega}$.

Proof: It suffices to check only the last part. Since from the first part $F_{\mathrm{A}}$ is written by the linear combination of $\left(\theta^{2 i-1}-\sqrt{-1} \theta^{2 i}\right) \wedge(\eta+\sqrt{-1} d t), F_{\mathbf{A}}$ is clearly orthogonal to $\tilde{\omega}$.

If the almost complex structure $J$ on $M \times \mathbf{R}$ is integrable, namely, the contact structure $\eta$ on $M$ is normal, then from (iii) of Proposition 7 the SU(2) connection $\mathbf{A}$ which associates with a $-\{(n-1) !\}^{-1}$-generalized monopole $(A, \Phi)$ induces a holomorphic vector bundle over $M \times \mathbf{R}$ equipped with an Einstein Hermitian bundle metric.

\section{FINAL REMARKS.}

To conclude this note, we give several remarks.

Pedersen and Poon, ${ }^{11}$ and Galicki and Poon ${ }^{6}$ gave another generalization of 3-dimensional magnetic monopole over $\mathbf{R}^{3 n}=\mathbf{R}^{3} \otimes \mathbf{R}^{n}$ by multitimes independent instantons on $\mathbf{R}^{4 n}$. However, our generalization is valid over any odd dimensional contact manifold, even though over the Eucliden space $\mathbf{R}^{\ell}, \ell>3$, with the natural contact form $\eta$ non-trivial solutions of our generalized Bogomolny equations are not yet obtained. An arbitrary compact semisimple Lie group can be taken as a gauge group $G$ which in this note we specialized as SU(2). For an arbitrary compact semisimple Lie group $G$ we impose the gauge invariant ansatz on Higgs fields. Actually we 
consider for this general case configurations satisfying the asymptotical decay conditions ( $\downarrow 2)$ and further require that the Higgs field at infinity $\Phi_{\infty}$ has the image sitting inside an adjoint action orbit in the Lie algebra so that $\Phi_{\infty}$ is regarded as a map from $\partial M_{\infty}$ to a homogeneous space $G / K$ ( $K$ is the isotropy subgroup for the orbit). See for this Itoh, ${ }^{12}$ Horváthy, and Rawnsley. ${ }^{4}$

So in this most general situation we observe the following topological phenomenon entirely different from the 3-dim original monopoles. For $M=\mathbf{R}^{2 n+1}, n \geqslant 2$ the boundary at infinity $\partial M_{\infty}$ is diffeomorphic to $S^{2 n}$ and the Higgs field at infinity $\Phi_{\infty}$ defines a class in $\pi_{2 n}(G / K)$ which happens to be trivial, e.g., $n=2$ and $G / K=\mathbf{C} P^{k}, k \geqslant 2$ so that this homotopy triviality might give a strict restriction on the topological invariant $p\left(\Phi_{\infty}\right)$.

\footnotetext{
'A. Jaffe and C. Taubes, Vortices and Monopoles (Birkhäuser, Boston, 1980).

${ }^{2}$ D. Groisser, Commun. Math. Phys. 93, 367 (1984).

${ }^{3} \mathrm{M}$. Atiyah and N. Hitchin, The Geometry and Dynamics of Magnetic Monopoles (Princeton University, Princeton, 1988).

${ }^{4}$ P. A. Horváthy and J. H. Rawnsley, Commun. Math. Phys. 96, 497 (1984).

${ }^{5}$ D. E. Blair, Contact Manifolds in Riemannian Geometry, Lecture Notes in Math. 509 (Springer-Verlag, Berlin, 1976).

${ }^{6}$ K. Galicki and Y. S. Poon, J. Math. Phys. 32, 1263 (1991).

${ }^{7}$ H. J. Kim, Ph. D. thesis, Berkeley, 1985.

${ }^{8}$ Y. Suh, Tsukuba J. Math. 14, 505 (1990).

${ }^{9}$ N. Manton, Nucl. Phys. B 135, 319 (1978).

${ }^{10} \mathrm{~S}$. Kobayashi, Differential Geometry of Complex Vector Bundles (Iwanami, Tokyo, 1987).

${ }^{1}$ H. Pedersen and Y. S. Poon, Commun. Math. Phys, 117, 569 (1986).

${ }^{12} \mathrm{M}$. Itoh, in Geometry and its Applications, edited by Nagano et al. (World Scientific, Singapore, 1993).
} 